\title{
Kombinierte Anwendung von Strahlentherapie und adjuvanter Therapie mit einem Mistelextrakt (Viscum album L.) zur Behandlung des oralen malignen Melanoms beim Hund: Eine retrospektive Studie
}

\author{
a Praxis 4 Pfoten, Port, Schweiz; \\ ${ }^{b}$ Animal Oncology and Imaging Center (AOI), Hünenberg, Schweiz; \\ ${ }^{c}$ Gesellschaft für klinische Forschung e.V. (GKF), Berlin, Deutschland; \\ ${ }^{\mathrm{d}}$ Forschungsinstitut für biologischen Landbau (FiBL), Frick, Schweiz
}

Uta von Bodungen ${ }^{a}$ Katja Ruess $^{b}$ Marcus Reif ${ }^{c} \quad$ Ulrike Biegel $^{d}$

\section{Schlüsselwörter \\ Orales Melanom $\cdot$ Hund $\cdot$ Mistelextrakt}

\section{Zusammenfassung}

Hintergrund: Orale maligne Melanome (OMM) des Hundes zeichnen sich durch schnelles Wachstum, lokale Invasion und hohe Metastasierungsraten aus. Extrakte auf Basis von Viscum album L. (VAE) werden zunehmend in der Krebstherapie sowohl in der Human- als auch in der Veterinärmedizin eingesetzt. Ziel unserer Studie war es zu untersuchen, inwieweit die adjuvante Therapie mit VAE eine therapeutische Option zur Behandlung von OMM ist. Besonderes Augenmerk galt dabei der Überlebenszeit und möglichen Nebenwirkungen. Tiere und Methoden: 26 Hunde mit OMM, die in einem der größten veterinäronkologischen Zentren der Schweiz allesamt eine Strahlentherapie erhielten (teilweise nach operativer Tumorresektion) wurden in die retrospektive Studie eingeschlossen: 18 Hunde wurden mit VAE behandelt $\left(1 \mathrm{ml}\right.$ VAE (Iscador $\left.{ }^{\circledR}\right)$ in ansteigenden Konzentrationen von 0,1 bis $20 \mathrm{mg} / \mathrm{ml}$ subkutan 3-mal pro Woche (VAE-Gruppe), 8 erhielten keine adjuvante Behandlung (Vergleichsgruppe). Wir verglichen die Größenentwicklung der OMM sowie die Überlebenszeit. Ergebnisse: Patienten mit Bestrahlung und adjuvanter VAE-Therapie zeigten mit 236 Tagen eine signifikant längere mediane Überlebenszeit im Vergleich zu Patienten mit Bestrahlung, aber ohne adjuvante VAE-Therapie (49 Tage; Log-Rank-Test: $p=0,0047$ ). Die VAE-Therapie verlängerte die Überlebenszeit um mehr als zwei Drittel (Hazard Ratio $(H R)=0,30,95 \%$-Konfidenzintervall (KI) 0,11-0,86; $p=0,024$ ), während ein höheres Tumorstadium gemäß UICC (Union internationale contre le cancer) einen statistischen Trend zur Verdopplung des Sterberisikos zeigte (UICC-Stadium III/IV vs. I/II: HR =2,12, 95\%-KI 0,88-5,12; $p=0,095$ ). Zwei Patienten zeigten milde Nebenwirkungen während der VAE-Behandlung. Einer der beiden zeigte 1 Tag lang ein selbstlimitiertes Fieber, bei dem anderen Patienten reduzierten wir die Dosis von einem konzentrierteren zu einem weniger konzentrierten VAE (Serie 0) aufgrund von Müdigkeit, die daraufhin verschwand. Schlussfolgerungen: VAE ist eine sichere, nebenwirkungsarme Behandlung und scheint sich positiv auf die Überlebenszeit von Hunden mit OMM auszuwirken. Somit ist dieser therapeutische Ansatz es wert, vermehrt bei der adjuvant zur Strahlentherapie eingesetzten Behandlung des OMM in Betracht gezogen zu werden. Die verglichenen Gruppen waren jedoch klein, divers und nicht konsistent hinsichtlich aller prognostischen Parameter. Eine prospektive Studie mit einer größeren Studienpopulation wäre daher von Interesse.

(c) 2017 The Author(s). Published by S. Karger GmbH, Freiburg

\section{KARGER}

Fax +497614520714
Karger

Open access

Keywords

Oral melanoma · Dog · Viscum album L. extract

\section{Summary}

Combination Therapy with Radiation and Adjuvant Mistletoe Extract (Viscum album L.) for the Treatment of Oral Malignant Melanoma in Dogs: A Retrospective Study

Background: Canine oral malignant melanomas (OMMs) are characterized by rapid growth, local invasion, and high metastatic rates. Viscum album L. extracts (VAE) are increasingly used in human but also in veterinary cancer treatment. The aim of our study was to investigate if an adjuvant therapy with VAE might be a potential treatment for OMM, with special regard to survival time and side effects. Animals and Methods: 26 dogs with OMM receiving radiation therapy (partly after surgery) in a larger Swiss veterinary oncological center were included in a retrospective study: 18 dogs were treated with VAE (VAE group: $1 \mathrm{ml}$ of VAE (Iscador ${ }^{\circledR}$ ) in increasing concentrations from 0.1 to $20 \mathrm{mg} / \mathrm{ml}$ was given subcutaneously); 8 dogs did not receive any adjuvant treatment (control group). We compared the development of OMM size and survival time. Results: Patients with radiation and adjuvant VAE therapy showed a significantly longer median survival time of 236 days compared to patients with exclusive radiation (49 days; log-rank test: $p=$ $0.0047)$. The VAE therapy prolonged the survival by more than twothirds (hazard ratio $(\mathrm{HR})=0.30,95 \%$ confidence interval $(\mathrm{Cl}) 0.11-0.86$; $p=0.024)$, while there was a statistical trend for an advanced staging according to the Union for International Cancer Control (UICC) to more than double the risk of death (UICC stage III/IV vs. I/II: HR $=2.12,95 \% \mathrm{CI}$ $0.88-5.12 ; p=0.095$ ). Two patients showed adverse effects of VAE: One patient showed 1 day of self-limited fever, and in the other case we had to reduce the dosage to a less concentrated VAE (series 0 ) because of fatigue, which then disappeared. Conclusions: VAE represents a safe treatment and seems to improve the survival time in dogs after radiation therapy of OMM, making it worthwhile for being taken into consideration as OMM therapy. However, the compared groups were small, highly diverse, and inconsistent in all prognostic parameters, so that a prospective study with a larger study population might be of interest. 


\section{Einleitung}

Das orale Melanom des Hundes verhält sich durch sein schnelles Wachstum, die lokale Infiltration und eine sehr hohe Metastasierungsrate hochmaligne. Die Patienten sterben ohne Therapie innerhalb kurzer Zeit an Metastasen. Deshalb ist neben der Chirurgie und Bestrahlung eine nebenwirkungsarme, systemische Therapie erforderlich [1-3]. Das orale maligne Melanom (OMM) ist der häufigste orale Tumor des Hundes [4]. Es wächst lokal invasiv und weist eine Metastasierungsrate von mehr als $80 \%$ auf [1-3]. Das Staging erfolgt nach Klassifikation unter Berücksichtigung der Tumorgröße, der Lymphknotenmetastasen und der Fernmetastasen (TNM $=$ Tumor/Nodule/Metastasen). Dieses TNM-System unterliegt den internationalen Vorgaben der Union internationale contre le cancer (UICC). Es werden folgende Stadien unterschieden [5]:

- TNM-UICC I: Tumoren $<2 \mathrm{~cm}$ Durchmesser, keine Lymphknoten- oder Fernmetastasen

- TNM-UICC II: Tumoren von 2 bis $<4 \mathrm{~cm}$, keine Lymphknoten- oder Fernmetastasen

- TNM-UICC III: Tumoren $\geq 4 \mathrm{~cm}$ und/oder Metastasen regionaler Lymphknoten

- TNM-UICC IV: Tumoren jede Größe, Lymphknoten- und/ oder Fernmetastasen

Typische Lokalisationen von Metastasen sind die regionalen Lymphknoten (in bis zu 74\% der Fälle) und die Lunge (in bis zu 67\%) [3].

Behandlungsstandard ist eine radikale chirurgische Resektion. In den häufigen Fällen von nicht resezierbaren Tumoren oder bei histologisch nachgewiesener unvollständiger chirurgischer Resektion empfiehlt sich eine Strahlentherapie, um das lokale Geschehen zu kontrollieren.

Die mediane Überlebenszeit für Hunde mit OMM nach Chirurgie liegt in den Stadien I, II und III bei ungefähr 17-18, 5 bzw. 3 Monaten [4].

Signifikant negative prognostische Faktoren sind ein fortgeschrittenes Stadium, die Tumorgröße, aber auch das Patientenalter und eine unvollständige chirurgische Resektion [6-12].

Die Kombination von Chirurgie und Strahlentherapie gestattet oft eine lokale Kontrolle. Die schnelle Metastasierung bleibt aber der lebenslimitierende Faktor und somit auch Ursache für die schlechte Prognose [13-15].

Bei einer Chemotherapie liegen die Gesamtansprechraten unter 30\% [3]. Deshalb sind neue, adjuvante Therapieoptionen nötig, die sich gezielt gegen den metastatischen Verlauf der Erkrankung richten.

\section{Adjuvante Therapie mit Extrakt aus Viscum album $L$.}

In der Humanmedizin konnten Augustin et al. [16] in einer retrospektiven Studie zu Patienten mit malignem Melanom TNMUICC II-III nach Misteltherapie eine signifikant geringere tumorbedingte Mortalität beobachten als bei einer Kontrollgruppe ohne Mistel (8,9\% nach 81 Monaten gegenüber 10,7\% nach 52 Monaten Beobachtungszeit). Das Intervall ohne Krankheitssymptome und das Überleben ohne Hirnmetastasen waren in der Gruppe, die ein
Viscum-album-L.-Extrakt (VAE) erhielt, ebenfalls signifikant besser [16]. Stumpf et al. [17] beschreiben in einer retrospektiven Studie, dass bei der Therapie von humanen Melanompatienten mit Misteltherapie keine relevanten Nebenwirkungen auftraten, wohingegen kein signifikanter Einfluss auf die Mortalität gezeigt werden konnte.

Auch in der Veterinärmedizin wird die Misteltherapie bei Krebserkrankungen eingesetzt und in verschiedenen Studien konnte deren Wirkung und gute Verträglichkeit gezeigt werden: Lefebvre et al. [18] behandelten das Sticker-Sarkom beim Hund unter üblicher Chemotherapie zusätzlich auch mit VAE-Therapie. Die VincristinDosis konnte signifikant reduziert werden. Außerdem wurde die Chemotherapie-assoziierte Leukopenie und die Rezidivbildung bei den VAE-Patienten deutlich reduziert. Christen-Clottu et al. [19] zeigten in ihrer randomisierten, placebokontrollierten Studie eine signifikant bessere Heilungsrate von VAE-behandelten Pferden mit equinem Sarkoid im Vergleich zur Placebogruppe. In einer Fallserie von Biegel et al. [20] verlängerte sich beim Fibrosarkom der Katze das krankheitsfreie Intervall nach Operation unter adjuvanter oraler Therapie mit einem Eichenmistelextrakt (Viscum quercus praeparatum Dilutio aquosa $0,1 \%$ ig) im Vergleich zu aktuellen Literaturangaben zu ausschließlich operierten Tieren.

Ziel der Studie war es, anhand retrospektiver Daten abzuschätzen, inwieweit bei Hunden, die aufgrund einer OMM-Diagnose (teilweise nach vorausgehender Operation) strahlentherapeutisch behandelt wurden, die adjuvante Therapie mit VAE a) gut verträglich ist und b) die Überlebenszeit der Patienten verlängert.

\section{Tiere und Methoden}

Alle Hunde mit histologisch gesichertem OMM-Befund, bei denen zwischen dem 1. Januar 2009 und dem 30. Juni 2014 im AOI Center in Hünenberg, einem der größten veterinäronkologischen Überweisungszentren der Schweiz, mit einer Strahlentherapie begonnen wurde $(n=26)$, wurden retrospektiv in die Studie eingeschlossen. Die Datengrundlage hinsichtlich der demografischen und prognostischen Faktoren sowie Angaben zur Größenentwicklung der Primärtumoren wurden den Aufzeichnungen des AOI Center entnommen. In der Regel wurden die Tiere 2-3 Wochen nach Ende der Strahlentherapie und anschließend alle 3 Monate zur Nachkontrolle einbestellt. Zur Ermittlung der Überlebenszeit wurden zwischen dem 1. Juli 2015 und dem 30. Oktober 2016 alle Tierarztpraxen, an die die Tiere rücküberwiesen wurden, telefonisch kontaktiert, um den Krankheitsverlauf und das Sterbedatum der Tiere zu ermitteln.

Die Bestrahlung erfolgte im AOI Center 4-mal im Abstand von 1 Woche mit 6-8 Gy. Patienten mit adjuvanter Melanomvakzine wurden ausgeschlossen. Von den 26 Hunden erhielten 18 eine adjuvante VAE-Therapie (VAEGruppe) und 8 keine adjuvante Therapie (Vergleichsgruppe). Die Misteltherapie begann in der Regel während der Phase der Bestrahlung oder kurz nach der letzten Bestrahlung. Die Entscheidung für oder gegen die Misteltherapie erfolgte aus nicht näher eruierbaren Gründen und dürfte eine individuelle Einzelfallentscheidung von Tierarzt und Tierbesitzer gewesen sein. 18 Hunde wurden in Abhängigkeit vom Ergebnis der klinischen Untersuchung zusätzlich vor der Bestrahlung operativ behandelt (Tab. 1; Online Supplementary Table; www. karger.com/?DOI=485743).

Angelehnt an die Anwendung in der Humanmedizin erhielten die Hunde der VAE-Gruppe einen standardisierten Extrakt der europäischen Weißbeerigen Mistel (Viscum album L.), Iscador ${ }^{\circledR}$, nach dem Behandlungsprotokoll der Viscumvet-Gruppe (www.viscumvet.org). In aufsteigender Dosierung von 0,01, $0,1,1,10$ und $20 \mathrm{mg} / \mathrm{ml}$ (sogenannte Serien) beginnend mit der Serie 0 wurde 
Tab. 1. Demografische und prognostische Angaben zur Studienpopulation

\begin{tabular}{lcccc}
\hline & VAE-Gruppe $^{\mathrm{a}}$ & \multicolumn{3}{c}{ Vergleichsgruppe $^{\mathrm{a}}$} \\
\hline Patienten & 18 & & 8 & \\
Alter, Jahre & 12,0 & $\pm 2,6$ & 11,4 & $\pm 2,1$ \\
Gewicht, kg & 24,8 & $\pm 14,7$ & 24,9 & $\pm 17,3$ \\
Geschlecht m gesamt & 7 & $38,9 \%$ & 5 & $62,5 \%$ \\
Davon mk & 2 & $11,1 \%$ & 4 & $50,0 \%$ \\
Geschlecht w gesamt & 11 & $61,1 \%$ & 3 & $37,5 \%$ \\
Davon wk & 4 & $22,2 \%$ & 3 & $37,5 \%$ \\
Chemotherapie & 1 & $5,6 \%$ & 0 & $0,0 \%$ \\
TNM-UICC I & 9 & $50,0 \%$ & 2 & $25,0 \%$ \\
TNM-UICC II & 1 & $5,6 \%$ & 1 & $12,5 \%$ \\
TNM-UICC III & 8 & $44,4 \%$ & 4 & $12,5 \%$ \\
TNM-UICC IV & 0 & $0,0 \%$ & 1 & $37,5 \%$ \\
Keine Metastasen & 10 & $55,6 \%$ & 3 & $75 \%$ \\
$\quad$ TNM-UICC I + II) & & & & \\
Chirurgie ja & 12 & $66,7 \%$ & 6 & \\
\hline
\end{tabular}

${ }^{a}$ Mittelwert \pm Standardabweichung bzw. absolute/relative Häufigkeit.

$\mathrm{VAE}=$ Extrakt aus Viscum album L.; $\mathrm{m}$ = männlich; $\mathrm{mk}=$ männlich kastriert; $\mathrm{w}=$ weiblich; $\mathrm{wk}$ = weiblich kastriert; TNM-UICC $=$ Tumor-Nodule-Metastasen-System entsprechend der internationalen Vorgaben der Union internationale contre le cancer. 3-mal pro Woche je 1 Ampulle subkutan appliziert. Je nach Reaktionslage des Hundes wurde nach 14 Ampullen und einer darauffolgenden 1-wöchigen Pause die Dosierung gesteigert. Bei 15 Hunden wurde Iscador ${ }^{\circledR} \mathrm{P}($ Pinus $=$ Kiefernmistel), bei 3 Hunden Iscador ${ }^{\circledR} \mathrm{M}$ (Malus = Apfelbaummistel) verabreicht. Die Hunde erhielten die Therapie als Dauertherapie bis an ihr Lebensende.

\section{Statistische Analyse}

Demografische, prognostische und Verlaufsangaben wurden als Mittel wert \pm Standardabweichung für intervallskalierte Daten, als Median für Überlebenszeitdaten bzw. als absolute/relative Häufigkeitstabellen für kategoriale Variablen berechnet. Die Überlebenszeit wurde definiert als Zeitspanne zwischen dem Tag der ersten Radiotherapie bis zum Tod bzw. andernfalls dem letzten Lebendkontakt des Patienten und getrennt für die beiden Behandlungsgruppen deskriptiv als Kaplan-Meier-Grafik dargestellt. Zum statistischen Vergleich wurden - neben der VAE-Therapie - die klinisch relevanten Parameter Alter, Geschlecht, Körpergewicht, Kastrationszustand, TNM-UICC-Status und vorherige Operation herangezogen. Mittels eines Cox Proportional Hazard-Regressionsmodells mit schrittweiser (stepwise) Variablenauswahl wurden die Parameter identifiziert, die in einer multivariablen Analyse einen signifikanten Einfluss auf das Überleben aufwiesen. Die Einflussstärke eines Parameters wurde als Verhältnis des Versterberisikos zwischen den untersuchten Gruppen dargestellt ( $\mathrm{Ha}-$ zard Ratio (HR)), dabei bedeutet HR = 1 keinen Unterschied, ein kleinerer/ größerer Wert jeweils ein kleineres/größeres Versterberisiko. Die statistischen Tests wurden 2-seitig bei einem Fehler erster Art von 5\% durchgeführt. Zusätzlich wurden 95\%-Konfidenzintervalle (95\%-KI) geschätzt.

In einer Sensitivitätsanalyse wurde zusätzlich mittels logistischer Regression ein Propensity Score berechnet, der die Unterschiede zwischen den beiden Studiengruppen hinsichtlich mehrerer Parameter in der statistischen Analyse mit berücksichtigen sollte [21]. Das Ergebnis der Hauptanalyse blieb dadurch jedoch qualitativ unverändert (Ergebnisse nicht gezeigt).

\section{Ergebnisse}

\section{Studienpopulation}

Die Studienpopulation hatte bei Diagnosestellung ein Durchschnittsalter von 12 Jahren in der VAE-Gruppe und von 11,4 Jahren in der Vergleichsgruppe. Die Studie enthält mit 14 Hündinnen und 12 Rüden eine ausgeglichene Geschlechtsverteilung, wobei die VAE-Gruppe anteilmäßig deutlich mehr Hündinnen enthält als die Kontrollgruppe (Tab. 1; Online Supplementary Table; www. karger.com/?DOI=485743).

Die Hunderassen waren sehr unterschiedlich, sowohl in der VAE-Gruppe (Labrador 3×, Dogge 1×, Dobermann 1×, Deutscher Schäferhund (DSH) 1×, DSH-Mischling 1×, Golden Retriever 1×, Irish Setter $1 \times$, Vorstehhund $1 \times$, Australian Shepherd $2 \times$, Mittelpudel $1 \times$, Cocker Spaniel $1 \times$, Dackel $2 \times$, Pekinese 1×, Mischling $1 \times$ ) als auch in der Vergleichsgruppe (Rottweiler $2 \times$, Golden Retriever $1 \times$, Mischling $3 \times$, Cocker Spaniel 1×, Yorkshire Terrier 1×). Auffallend häufig waren große Hunde mit nahezu identischem Durchschnittsgewicht von 24,8 kg (VAE-Gruppe) und 24,9 kg (Vergleichsgruppe) betroffen. In der VAE-Gruppe finden sich mit 55,6\% mehr Tumorstadien ohne Metastasen als in der Vergleichsgruppe (37,5\%). Die VAE-Gruppe enthält $66,7 \%$ operierte Patienten, die Vergleichsgruppe $75 \%$.

\section{Entwicklung der Tumorgröße}

Von 18 VAE-therapierten Hunden zeigten 6 Patienten (33,3\%) eine komplette, 7 Patienten (38,9\%) eine partielle Remission des Tumors (> 30\% Tumorreduktion) und 4 Patienten (22,2\%) einen stabilen Krankheitsverlauf (<30\% Tumorreduktion; Tab. 2).

In der Vergleichsgruppe zeigte sich in dieser Untersuchung keine komplette Remission, bei 6 Patienten (75\%) eine partielle Remission des Tumors (> 30\%) und bei 1 Patienten (12,5\%) ein stabiler Krankheitsverlauf ( $<30 \%$ Tumorreduktion). Angaben zum Krankheitsverlauf von 1 Patienten in der VAE- und von 1 Patienten in der Vergleichsgruppe waren nicht eruierbar.

\section{Nebenwirkungen}

In der Mistelbehandlungsgruppe zeigten 2 von 18 Patienten Nebenwirkungen. Ein Patient hatte 1-malig Fieber 1 Tag nach der 
Tab. 2. Remission caniner OMM radiotherapeutisch behandelter Hunde nach adjuvanter Therapie mit VAE im Vergleich zu nicht adjuvant behandelten Hunden desselben veterinäronkologischen Therapiezentrums

\begin{tabular}{lcccr}
\hline Kennzahl & VAE-Gruppe & \multicolumn{2}{c}{ Vergleichsgruppe } \\
\hline $\begin{array}{l}\text { Gesamtanzahl Hunde, N } \\
\text { Komplette Remission, } \mathrm{n}\end{array}$ & 18 & $33 \%$ & 8 & $0 \%$ \\
$\begin{array}{l}\text { Partielle Remission } \\
\quad(>30 \% \text { Reduktion), } \mathrm{n}\end{array}$ & 7 & $39 \%$ & 6 & $75 \%$ \\
$\begin{array}{l}\text { Stabile Krankheit (keine } \\
\quad \text { bis }<30 \% \text { Reduktion), n }\end{array}$ & 4 & $22 \%$ & 1 & $13 \%$ \\
$\begin{array}{l}\text { Angaben fehlend, n } \\
\text { OMM = Orales malignes Melanom; VAE = Extrakt aus Viscum album } \mathrm{L} .\end{array}$ & $13 \%$ \\
\hline
\end{tabular}

\section{Diskussion}

Die Patienten der VAE-Gruppe überlebten signifikant länger als die der Kontrollgruppe. Der Log-Rank-Test zeigt ein statistisch signifikant längeres Überleben in der VAE-Gruppe ( $\mathrm{p}=$ 0,047). Die Überlebenszeiten streuen allerdings sehr breit und die beiden Behandlungsgruppen unterscheiden sich in mehreren $\mathrm{Pa}$ rametern deutlich. Die Zuteilung auf die beiden Behandlungsgruppen erfolgte auch nicht zufällig, sondern auf Basis der vermutlich gemeinsamen Entscheidung von Tierarzt und Tierbesitzer, was allerdings bei retrospektiven Studien dieser Art nicht zu verhindern ist und auch auf andere Studien zum caninen OMM zutrifft $[11,14]$.

In der VAE-Gruppe kam sowohl Pinien- als auch Apfelbaummistel zur Anwendung, wobei erfahrungsgemäß beim OMM primär die Pinienmistel zum Einsatz empfohlen wird. Die Wahl der Mistelart bei der VAE-Therapie dürfte dabei in unserem Datensatz derselben therapeutischen Freiheit und Variation unterliegen wie die Wahl adjuvant applizierter Chemotherapeutika in anderen retrospektiven Studien [14].

Der Unterschied zwischen den beiden Gruppen könnte partiell durch die verschiedenen Tumorstadien bedingt sein. In der VAEGruppe zeigen 55,6\% der Hunde Tumorstadien ohne Metastasen, in der Vergleichsgruppe sind es mit 37,5\% deutlich weniger. Außerdem erhielt 1 Patient in der VAE-Gruppe eine Chemotherapie (Doxorubicin) wegen eines zusätzlichen Mammatumors. Dies könnte ebenfalls zum besseren Abschneiden dieser Gruppe beigetragen haben.

Die Anzahl an operierten Tieren beträgt andererseits in der VAE-Gruppe 66,7\% $(n=12 / 18)$ gegenüber $75 \%(n=6 / 8)$ in der Vergleichsgruppe, was wiederum eher die Kontrollgruppe bevorteilt. Die durchgeführte multivariable statistische Analyse ist geeignet, diese Unterschiede zwischen den Behandlungsgruppen zu berücksichtigen. Allerdings sind die Fallzahlen zu gering, um eine definitive Aussage bezüglich der wirklichen Einflussstärken aller klinisch relevanten Parameter treffen zu können. Die statistischen Methoden der Variablenselektions- und Propensity Score-Analyse wurden explizit aufgrund der relativ geringen Fallzahl gewählt und waren darauf angelegt, die Datenheterogenität und die durch die fehlende Randomisierung bedingten Unterschiede zwischen den beiden Studiengruppen weitestgehend zu berücksichtigen, wie für Beobachtungsstudien empfohlen 
Tab. 3. Adjuvante Therapie caniner OMM radiotherapeutisch behandelter Hunde: Vergleich eigener Daten zur adjuvanten Therapie mit VAE mit aktuellen Angaben aus der Literatur

\begin{tabular}{|c|c|c|c|c|c|c|}
\hline Studie & Staging & Gruppen & & $\mathrm{N}$ & MST, Tage & $\mathrm{p}$ \\
\hline \multirow{2}{*}{$\begin{array}{c}\text { Cancedda et al., } \\
2014 \text { [23] }\end{array}$} & I (1), II (1), III (10), IV (3) & Kontrolle & Bestrahlung & 15 & 192 & \multirow[t]{2}{*}{0,09} \\
\hline & I (1), II (2), III (8), IV (1) & Behandlung & Bestrahlung + Chemo & 12 & 401 & \\
\hline $\begin{array}{l}\text { Freeman et al., } \\
\qquad 2003 \text { [11] }\end{array}$ & I (22), II (14), III (3) & keine Kontrollgruppe & $\begin{array}{l}\text { OP, plus Chemo und } \\
\text { Bestrahlung }\end{array}$ & 39 & 363 & $\begin{array}{l}\text { keine } \\
\text { Angabe }\end{array}$ \\
\hline $\begin{array}{l}\text { Herzog et al., } \\
\qquad 2013 \text { [24] }\end{array}$ & ausschließlich Stage IV & keine Kontrollgruppe & $\begin{array}{l}\text { Bestrahlung (teilweise } \\
\text { OP) }+ \text { Vakzine }\end{array}$ & 9 & 154 & $\begin{array}{l}\text { keine } \\
\text { Angabe }\end{array}$ \\
\hline \multirow[t]{2}{*}{$\begin{array}{l}\text { Murphy et al., } \\
2005 \text { [12] }\end{array}$} & \multirow{2}{*}{$\begin{array}{l}\text { I (3), II (7), III (2), } \\
\text { unbekannt (1) } \\
\text { I (2), II (7), III (5), } \\
\text { unbekannt (1) }\end{array}$} & Kontrolle & Bestrahlung & 13 & 307 & \multirow[t]{2}{*}{$\begin{array}{l}\text { nicht } \\
\text { signifikant }\end{array}$} \\
\hline & & Behandlung & $\begin{array}{l}\text { Bestrahlung + } \\
\text { Carboplatin }\end{array}$ & 15 & 286 & \\
\hline \multirow{6}{*}{$\begin{array}{l}\text { Proulx et al., } \\
2003[14]\end{array}$} & \multirow[t]{6}{*}{ keine Angabe } & \multirow[t]{4}{*}{ alle } & & 140 & 210 & \multirow[t]{4}{*}{0,67} \\
\hline & & & Bestrahlung (30 Gy) & 69 & 207 & \\
\hline & & & Bestrahlung (36 Gy) & 54 & 213 & \\
\hline & & & Bestrahlung (> 45 Gy) & 17 & 111 & \\
\hline & & Kontrolle & Bestrahlung & 60 & $241^{\mathrm{a}}$ & 0,89 \\
\hline & & Behandlung & Bestrahlung + Chemo & 80 & $210^{\mathrm{b}}$ & \\
\hline \multirow[t]{2}{*}{ Eigene Daten } & I (2), II (1), III (4), IV (1) & Kontrolle & $\begin{array}{l}\text { Bestrahlung } \\
\text { (teilweise OP) }\end{array}$ & 8 & 49 & \multirow[t]{2}{*}{0,0047} \\
\hline & I (9), II (1), III (8), IV (0) & Behandlung & $\begin{array}{l}\text { Bestrahlung (teilweise } \\
\mathrm{OP})+ \text { Viscum album }\end{array}$ & 18 & 236 & \\
\hline \multicolumn{7}{|l|}{$\begin{array}{l}\text { a7,9 Monate. } \\
\text { b6,9 Monate. } \\
\text { OMM = Orales }\end{array}$} \\
\hline
\end{tabular}

[22]. Auch wenn sicherlich nicht alle relevanten Parameter identifiziert werden konnten, ist die Identifizierung des bekannten Einflusses des UICC-Stadiums auf das Überleben eine Bestätigung für die Validität des Befundes eines relevanten Einflusses der Misteltherapie.

In den vergangenen Jahren setzten sich nur wenige Studien mit der zur Bestrahlung adjuvanten systemischen Therapie des caninen oralen Melanoms auseinander, mit teilweise unbefriedigenden Ergebnissen. Wie auch in unserer Untersuchung ging der Bestrahlung teilweise eine chirurgische Behandlung voraus [11, 12, 14, 23, 24] (Tab. 3).

Zwei Studien beschrieben lediglich die Ergebnisse der adjuvanten Therapie. Eine ausschließlich mit Bestrahlung behandelte Kontrollgruppe fehlt diesen Studien [11, 24]: Herzog et al. [24] zeigten bei Patienten mit Bestrahlung und adjuvanter Melanomvakzinetherapie eine mediane Überlebenszeit von 161 Tagen. Bei diesen 9 Patienten handelte es sich um Patienten mit OMM-Stadium IV [24]; im Vergleich zu unserer Studienpopulation (mehrheitlich TNM-UICC I-III) waren diese Hunde wesentlich stärker von der Tumorerkrankung betroffen. Die retrospektive Studie von Freeman et al. [11] präsentierte eine mediane Überlebenszeit von 363 Tagen bei unvollständig resezierten OMM nach Bestrahlung und 60 min zuvor verabreichter Chemotherapie mit Cisplatin oder Carboplatin. Hier erfolgte eine Radiochemotherapie, in unserer Studie jedoch eine konventionelle Radiotherapie. Darüber hin- aus wiesen mehr als 50\% der Hunde dieser Studie ein TNM-UICC von I auf.

Die 3 verbleibenden Studien verglichen adjuvante Chemotherapie und Bestrahlung mit ausschließlicher Bestrahlung: Murphy et al. [12] zeigten in einer - hinsichtlich der Studienpopulation mit unserer Untersuchung vergleichbaren - Studie keinen signifikanten Unterschied zwischen den Überlebenszeiten der Melanompatienten unter Radiotherapie verglichen mit kombinierter Radio- und Carboplatin-Therapie. Jedoch wurden im Vergleich zu unseren Ergebnissen mit 307 Tagen (Radiotherapie) und 286 Tagen (Radiound Carboplatin-Therapie) relativ lange mediane Überlebenszeiten beobachtet. $60 \%$ der Patienten beider Gruppen wiesen keine Metastasen auf, was als positiver prognostischer Faktor diskutiert werden kann.

Proulx et al. [14] fanden keinen signifikanten Unterschied der Überlebenszeiten bei 3 verschiedenen Radiotherapieprotokollen. Hier wurde in der Studienpopulation eine mediane Überlebenszeit von 7 Monaten beobachtet. Diskutiert wurde ein Zusammenhang der Überlebenszeit mit Risikofaktoren wie Tumorgröße, Tumorlokalisation und Grad der Knochenlyse, was jeweils als negativer prognostischer Faktor diskutiert wurde. Auch in dieser Studie hatte die systemische adjuvante Chemotherapie keinen Einfluss auf die Überlebenszeit im Vergleich zur reinen Radiotherapie. Eine Studie von 2014 mit ebenfalls vergleichbarer Studienpopulation zeigte keinen signifikanten Vorteil der adjuvanten Chemotherapie [23]. 
Die aktuell verfügbare wissenschaftliche Literatur lässt weder die Chemotherapie noch die Vakzine [4, 25] als über jeden Zweifel erhabene adjuvante Therapie zur Strahlentherapie des caninen oralen Melanoms erscheinen.

Wenngleich unsere Ergebnisse anhand retrospektiver Daten zweier nicht einheitlicher Studiengruppen gewonnen wurden, was bei retrospektiven Daten und verhältnismäßig geringen Fallzahlen jedoch oft unvermeidbar ist, kann zusammenfassend erklärt werden, dass a) die Verträglichkeit der Misteltherapie bei allen behandelten Patienten sehr gut war und b) die mit Mistelextrakt behandelte Gruppe im Vergleich zur reinen Radiotherapie eine längere Überlebenszeit zeigte. Wie in der Literatur beschrieben scheint auch bei unserer Studienpopulation die Prognose umso günstiger $\mathrm{zu}$ sein, je kleiner der Tumor ist und je weniger Metastasen vorhanden sind. Das heißt, je früher das OMM erkannt und mit Chirurgie, Radiotherapie und systemischer adjuvanter Therapie behandelt wird, desto länger ist die Überlebenszeit.

Eine zukünftige prospektive, randomisierte, placebokontrollierte Studie - idealerweise mit vergleichbarerer Verteilung aller Tumorstadien auf die Gruppen - zur Wirksamkeit von VAE beim OMM beim Hund erscheint wünschenswert.

\section{Schlussfolgerung}

Wenngleich die geringe Fallzahl und der bestehende Gruppenunterschied hinsichtlich potenzieller weiterer Einflussfaktoren auf das Therapieergebnis die Aussagekraft des geringeren Sterberisikos in der VAE- im Vergleich zur Vergleichsgruppe einschränken, sollte die adjuvante systemische Therapie mit VAE vermehrt in Betracht gezogen werden, nicht zuletzt auch vor dem Hintergrund einer von der systemischen Chemotherapie ausgehenden erheblichen Belastung des Hundes, seiner Besitzer und der Umwelt.

\section{Online Supplementary Material}

Online Supplementary Table To access the supplementary table, please refer to $w w w$.karger.com/?DOI $=485743$.

\section{Disclosure Statement}

Diese Arbeit wurde durch den Verein für Krebsforschung (Arlesheim, Schweiz) unterstützt.

\section{Literatur}

1 Bostock DE: Prognosis after surgical excision of canine melanoma. Vet Pathol 1979;16:32-40.

2 Smith SH, Goldschmidt MH, McManus PM: A comparative review of melanocytic neoplasms. Vet Pathol 2002;39:651-678.

3 Liptak JM, Withrow SJ: Cancer of the gastrointestinal tract, oral tumors; in Vail DM (ed): Withrow and MacEwen's Small Animal Clinical Oncology, ed 5. St. Louis, Saunders Elsevier, 2013, pp 381-389.

4 Bergman PJ: Canine oral melanoma. Clin Tech Small Anim Pract 2007;22:55-60.

5 Owen LN: TNM classification of tumors in domestic animals. Geneva, World Health Organisation, 1980.

6 Boston SE, Lu X, Culp WT, Montinaro V, Romanelli G, Dudley RM, Liptak JM, Mestrinho LA, Buracco P: Efficacy of systemic adjuvant therapies administered to dogs after excision of oral malignant melanomas: 151 cases (2001-2012). J Am Vet Med Assoc 2014;245:401-407.

7 Kosovsky JK, Matthiesen DT, Marretta SM, Patnaik AK: Results of partial mandibulectomy for the treatment of oral tumors in 142 dogs. Vet Surg 1991;20: 397-401.

8 Wallace J, Matthiesen DT, Patnaik AK: Hemimaxillectomy for the treatment of oral tumors in 69 dogs. Vet Surg 1992;21:337-341.

9 Bateman KE, Catton PA, Pennock PW, Kruth SA: 0-721 radiation therapy for the treatment of canine oral melanoma. J Vet Intern Med 1994;8:267-272.

10 Blackwood L, Dobson JM: Radiotherapy of oral malignant melanomas in dogs. J Am Vet Med Assoc 1996; 209:98-102.

11 Freeman KP, Halm KA, Harris FD, King GK: Treatment of dogs with oral melanoma by hypofractionated radiation therapy and platinum-based chemotherapy (1987-1997). J Vet Intern Med 2003;17:96-101.
12 Murphy S, Hayes AM, Blackwood L, Maglennon G, Pattinson H, Sparkes AH: Oral malignant melanoma the effect of coarse fractionation radiotherapy alone or with adjuvant carboplatin therapy. Vet Comp Oncol 2005;3:222-229.

13 Rassnick KM, Ruslander DM, Cotter SM, Al-Sarraf R, Bruyette DS, Gamblin RM, Meleo KA, Moor AS: Use of carboplatin for treatment of dogs with malignant melanoma: 27 cases (1989-2000). J Am Vet Med Assoc 2001;218:1444-1448

14 Proulx DR, Ruslander DM, Dodge RK, Hauck ML, Williams LE, Horn B, Price GS, Thrall DE: A retrospective analysis of 140 dogs with oral melanoma treated with external beam radiation. Vet Radiol Ultrasound 2003;44:352-359.

15 Boria PA, Murry D, Bennett PF, Glickman NW, Snyder PW, Merkel BL, Schlittler DL, Mutsaers A, Thomas RM, Knapp DW: Evaluation of cisplatin combined with piroxicam for the treatment of oral malignant melanoma and oral squamous cell carcinoma in dogs. J Am Vet Med Assoc 2004;224:388-394.

16 Augustin M, Bock PR, Hanisch J, Karasmann M, Schneider B: Safety and efficacy of the long-term adjuvant treatment of primary intermediate- to high-risk malignant melanoma (UICC/AJCC stage II and III) with a standardized fermented European mistletoe (Viscum album L.) extract. Results from a multicenter, comparative, epidemiological cohort study in Germany and Switzerland. Arzneimittelforschung 2005;55:38-49.

17 Stumpf C, Rosenberger A, Rieger S, Tröger W, Schietzel M, Stein GM: Retrospective study of malignant melanoma patients treated with mistletoe extracts. Forsch Komplementarmed Klass Naturheilkd 2003;10: 248-255.
18 Lefebvre G, Bonamin LV, Oliveira CM: Tratamento de tumor venéreo transmissivel (tvt) canino utilizando Viscum album em associação à quimioterapia [Treatment of transmissible venereal tumor (tvt) in dogs with Viscum album (mistletoe) associated to chemotherapy]. Clin Vet (Milano) 2007;70:78-86.

19 Christen-Clottu O, Klocke P, Burger D, Straub R, Gerber $\mathrm{V}$ : Treatment of clinically diagnosed equine sarcoid with a mistletoe extract (Viscum album austriacus). J Vet Intern Med 2010;24:1483-1489.

20 Biegel U, Klocke P, Ruess-Melzer K, Kaser-Hotz B: Ein Mistelextrakt (Viscum album) als ergänzendes Therapiekonzept beim felinen Fibrosarkom [Mistletoe extracts (Viscum album) as an adjuvant therapy concept in feline fibrosarcoma]; in Scheer R, et al (eds): Die Mistel in der Tumortherapie 3. Essen, KVC-Verlag, 2013, pp 475-485

21 Rosenbaum PR, Rubin DB: The central role of the propensity score in observational studies for causal effects. Biometrika 1983;70:41-55.

22 Kreienbrock L, Pigeot I, Ahrens W: Epidemiologische Methoden, ed 5. Berlin/Heidelberg, Springer, 2012.

23 Cancedda S, Rohrer Bley C, Aresu L, Dacasto M, Leone VF, Pizzoni S, Gracis M, Marconato L: Efficacy and side effects of radiation therapy in comparison with radiation therapy and temozolomide in the treatment of measurable canine malignant melanoma. Vet Comp Oncol 2016;14:e146-e157.

24 Herzog H, Buchholz J, Ruess-Melzer K, Lang J, KaserHotz B: Kombinierte Anwendung von Strahlentherapie und DNS Tumor Vakzine zur Behandlung des oralen malignen Melanoms beim Hund: Eine Pilotstudie. Schweiz Arch Tierheilkd 2013;155:135-142.

25 Atherton MJ, Morris JS, McDermott MR, Lichty BD Cancer immunology and canine malignant melanoma: a comparative review. Vet Immunol Immunopathol 2016;169:15-26. 